\title{
MICROMACHINED MAGNETOSTATIC WAVE RESONATORS
}

\author{
George Sajin $^{(*)}$, Romolo Marcelli ${ }^{(* *)}$ \\ (*) National Research and Development Institute for Microtechnologies \\ PO Box 38-160, 72996, Bucharest, Romania. E-mail: gsajin@imt.ro \\ ${ }^{(* *)} \mathrm{M}^{2} \mathrm{~T}$ - Microwave Microsystems Technology, CNR-IMM, Rome Section \\ Via del Fosso del Cavaliere 100, 00133 Rome, Italy. E-mail: r.marcelli@psm.rm.cnr.it
}

\begin{abstract}
A frequency tunable magnetostatic wave (MSW) straight edge resonator (SER) made by a YIG film has been used as a selective frequency component in a micromachined resonating filter. $S$ parameters have been measured at different DC magnetic bias fields, with a frequency tunability between $2 \mathrm{GHz}$ and $6 \mathrm{GHz} c a$. An improvement of the performances for the SERs excited by micromachined microstrip transducers has been clearly demonstrated. Moreover, the utilization of silicon membranes to support MSW-SERs offers important openings toward the integration of magnetostatic wave devices with micromachined structures.
\end{abstract}

\section{INTRODUCTION}

Planar magnetostatic wave (MSW) technology is well known for providing frequency tunable filters and oscillators for linear and nonlinear microwave signal processing [1 - 4].

The size of MSW resonators is usually chosen to fulfill the conditions of good electrical matching and frequency selectivity of the resonator itself. MSW resonators in band-pass configurations are placed between two microstrip transducers with side-coupling for both the input and the output microstrip. The two microstrip transducers used for the excitation of the SER have to be properly designed to optimize the transmission characteristics and to reduce as much as possible the crosstalk effect. To fulfil such a condition, high impedance transducers can be used in the coupling region. In this paper, two configurations have been studied: (i) a bulk one with the two microstrips realized on a silicon substrate as discussed above, and (ii) a micromachined one with transducers having the same geometry but suspended on a membrane obtained by silicon substrate etching.

\section{DEVICE CONSTRUCTION}

In our experiment we have used two kinds of substrate: a $4000 \Omega . \mathrm{cm}, 400 \mu \mathrm{m}$ thick bulk silicon and an anisotropically etched silicon substrate to obtain a $50 \mu \mathrm{m}$ thick membrane. Silicon permittivity was $\varepsilon=11.7$ enabling a $w_{0}=500 \mu \mathrm{m}$ feeding microstrip lines in order to obtain a $50 \Omega$ impedance at the device ports. In the coupling region the transducers width/length dimensions were $50 \mu \mathrm{m} / 4$ $\mathrm{mm}$, to minimize the crosstalk effect. The transition between transducer lines and feeding microstrip lines were designed to have a progressive impedance change to provide a large band operation for the SER. $\mathrm{Cr} / \mathrm{Au}$ followed by $\mathrm{Au}$ electroplating was used for the microstrip transducers, up to a $1.5 \mu \mathrm{m}$ of thickness [5]. For device construction see, also, Fig.1 [6].

Two rectangular samples of epitaxial YIG/GGG layer, supported by silicon bulk or by silicon membrane, were used. Dimensions were: sample \#1: $4 \times 0.5 \times 0.32 \mathrm{~mm}^{3}$ and sample \#2: $4 \times 0.4 \times 0.32$ $\mathrm{mm}^{3}$. The same YIG/GGG chips were used in all experiments. In both samples the thickness of GGG support substrate was $0,30 \mathrm{~mm}$ and the thickness of active YIG layer was $0,020 \mathrm{~mm}$. These resonator structures were mounted on a suitable microstrip transducers test fixture. The 
microprocessed silicon substrate, the resonator structure and the test fixture are presented in Fig.1 (a), (b) and (c).

\section{EXPERIMENTAL RESULTS AND FULL RANGE ANALYSIS OF MSW-SER.}

In what follows, the measurements performed on the bulk and on the micromachined MSW-SER are presented. The resonators were biased by means of a DC magnetic field whose strength was changed to provide frequency tunability from $2 \mathrm{GHz}$ to $6 \mathrm{GHz}$ ca.. The $\mathrm{S}_{21}$ parameter was recorded to compare the performances of the bulk and micromachined structures by using two YIG resonators having different sizes. The frequency sweep domain was between $2 \mathrm{GHz}$ and $5 \mathrm{GHz}$ for silicon bulk resonators and between $2 \mathrm{GHz}$ and $6.5 \mathrm{GHz}$ for silicon membrane resonators

In Fig. 2 and Fig. 3 the measurements of the two YIG samples used on the bulk substrate are shown. The level of losses is quite high, close to $-20 \mathrm{~dB}$, and a high order mode appears not far from the main one with other lower intensity modes. The rejection ratio (RR) is greater than $20 \mathrm{~dB}$. The operative range is between 2.5 and $4.5 \mathrm{GHz}$, approximately. In Fig.3 similar results are obtained, when sample \#2 is investigated. Actually, the losses are still high, but there is only one high order mode which enters the spectrum and it is depressed $10 \mathrm{~dB}$ ca. with respect to the main mode.

Passing to the micromachined configuration, whose performances are shown in Fig.4 and in Fig.5, two main differences can be seen as compared to the bulk one. First of all, the $50 \Omega$ matching condition for the microstrips is valid also in the coupling region, and this solution helps the losses level, wich is now less than $10 \mathrm{~dB}$ in $\mathrm{S}_{21}$. On the other hand, the crosstalk is still under control, with an isolation better than $30 \mathrm{~dB}$. Secondly, the micromachining of the Si wafer has caused a shift of the operative frequencies available for the resonator, as expected from a decrease of the effective permittivity for the exploited structure, and a widening of the operative range.

\section{CONCLUSIONS}

Bulk and micromachined magnetostatic wave resonators have been characterized and the enhancement of the electrical performances for the micromachined structure with respect to the bulk one was demonstrated. Four SER configurations, two on a silicon bulk substrate and other two on silicon membrane, were measured. The obtained results demonstrate both: (i) the possibility to combine the MSW technology with the micromachining one, and (ii) the improvement obtained by the resonators supported on silicon membrane, with a decrease of $18 \mathrm{~dB}$ ca. in the insertion losses.

\section{ACKNOWLEDGEMENT}

Work partially funded by the Italy-Romania Protocol on Scientific and Technological Cooperation.

\section{REFERENCES}

[1] P. Kaboš and V.S. Stalmachov: "Magnetostatic Wave and Their Applications", Chapman \& Hall, London (1994).

[2] R. Marcelli and P. De Gasperis (Editors): “Advanced Linear and Non-linear Microwave Signal Processing by means of Magnetostatic Wave Devices", Research Signpost, Trivandrum, India (1996).

[3] R. Marcelli and S.A. Nikitov (Editors): "Nonlinear Microwave Signal Processing: Towards a New Range of Devices. Nonlinear Microwave Magnetic and Magnetooptic Information Processing”; Kluwer Academic Publishers, Dordrecht, The Netherlands (1996). 
[4] R. Marcelli, E. Andreta, G. Bartolucci, M. Cicolani, and A. Frattini: “A Magnetostatic Wave Oscillator for Data Relay Satellite”, IEEE Trans. On Magnetics, Vol.36, No.5, pp. (2000).

[5] G. Sajin, V. Avramescu, M. Dragoman, R. Marcelli: "Magnetostatic wave transducers on silicon wafers and on silicon membranes", Proceed. of International Semiconductor Conference, CAS'98, Sinaia, Romania, pp.635-638 (1998).

[6] G. Sajin, E. Matei, R. Marcelli: "Microwave Tunable Straight Edge Resonator on Silicon Membrane", Proceedings of GAAS (Gallium Arsenide Applications Symposium), CNIT, La Defense, Paris, 2-3 October, pp.373-376 (2000).

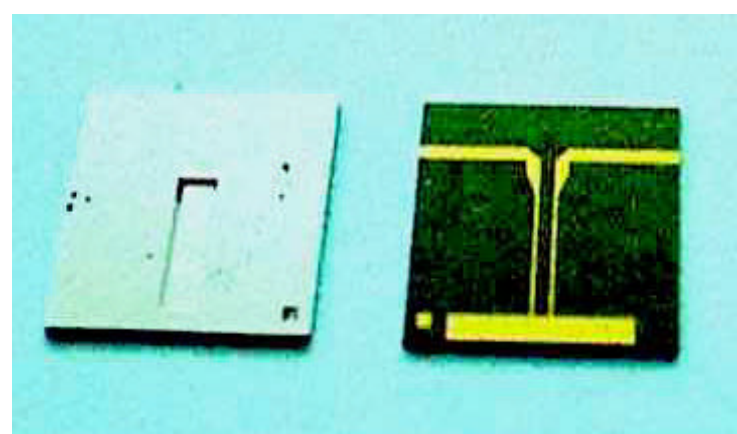

(a)

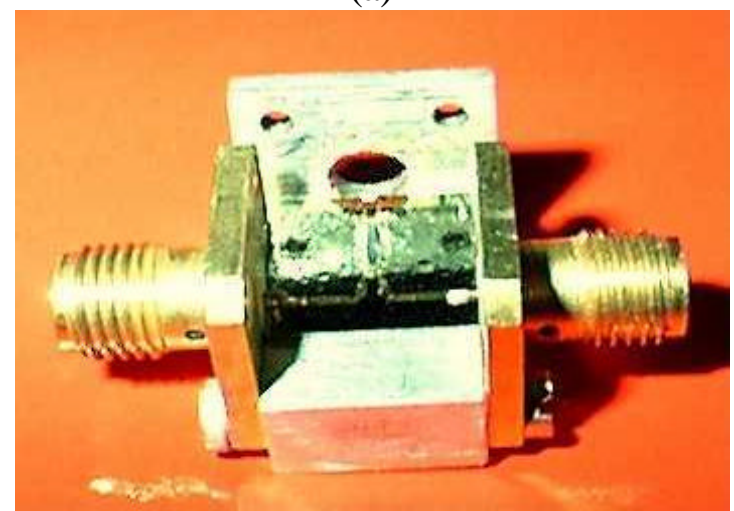

(c)

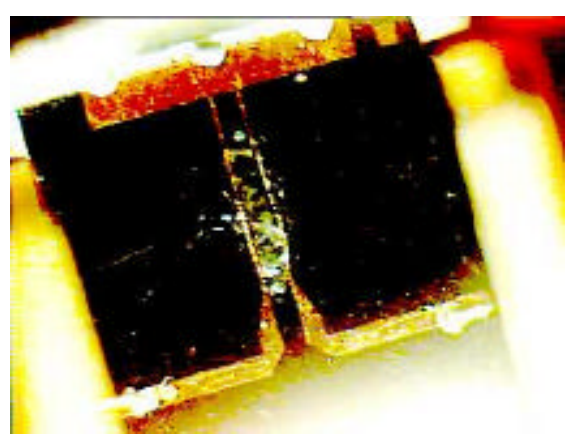

(b)

Fig.1. (a) The microprocessed silicon substrate. Back side: microprocessed membrane. Front side: microstrip transducers on silicon membrane. (b) The resonator structure, (c) Microwave test fixture.

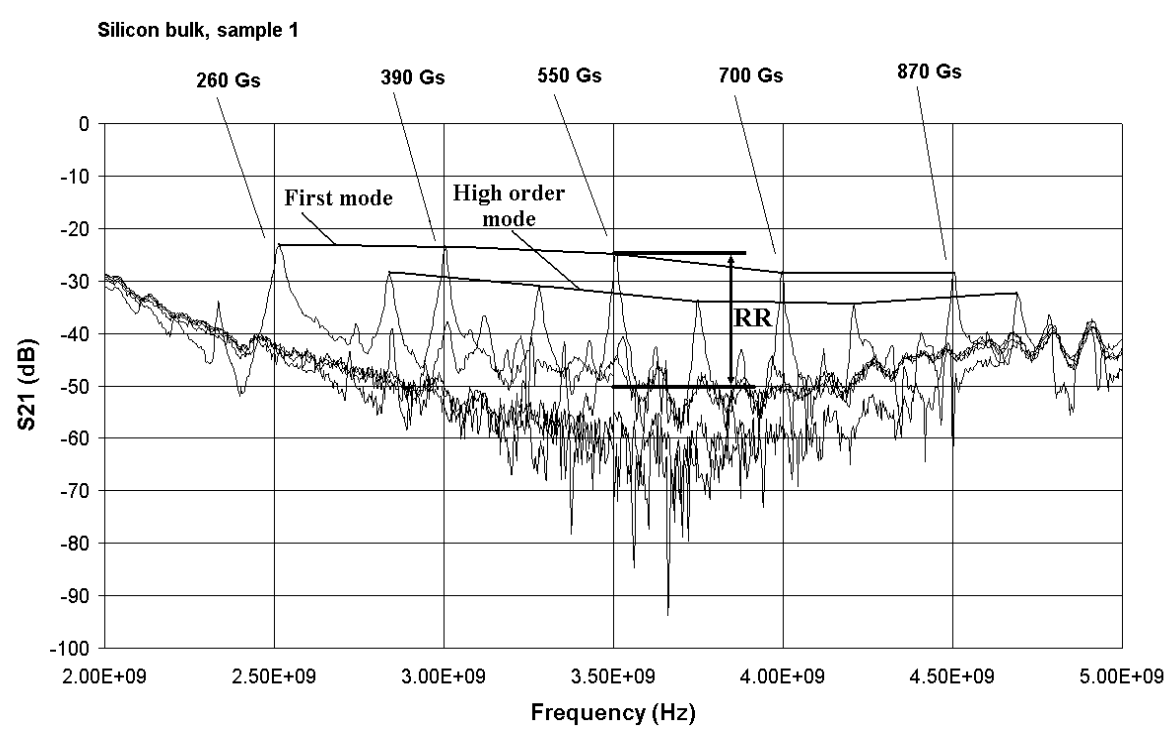

Fig. 2. Transmission characteristics for the bulk silicon MSW-SER, sample \#1. The strength of the dc field is given close to each resonance peak for the main mode. 


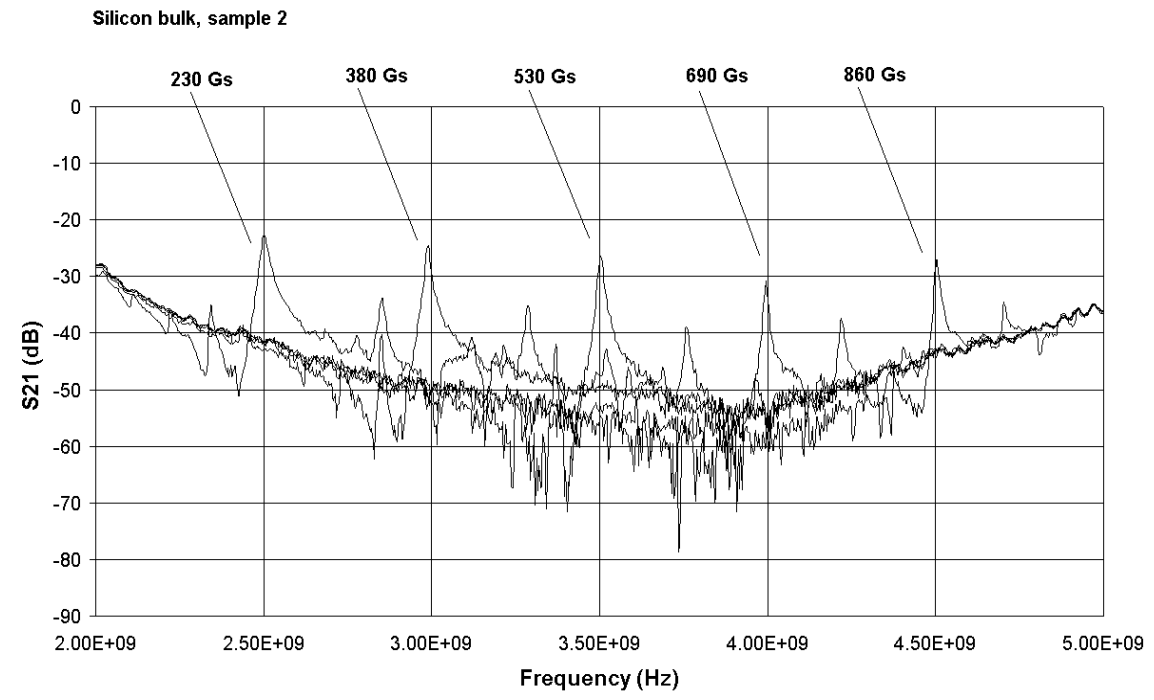

Fig. 3. Transmission characteristics for the bulk silicon MSW-SER, sample \#2. An improvement in the selectivity is recorded because of the different dimensions of the resonator.

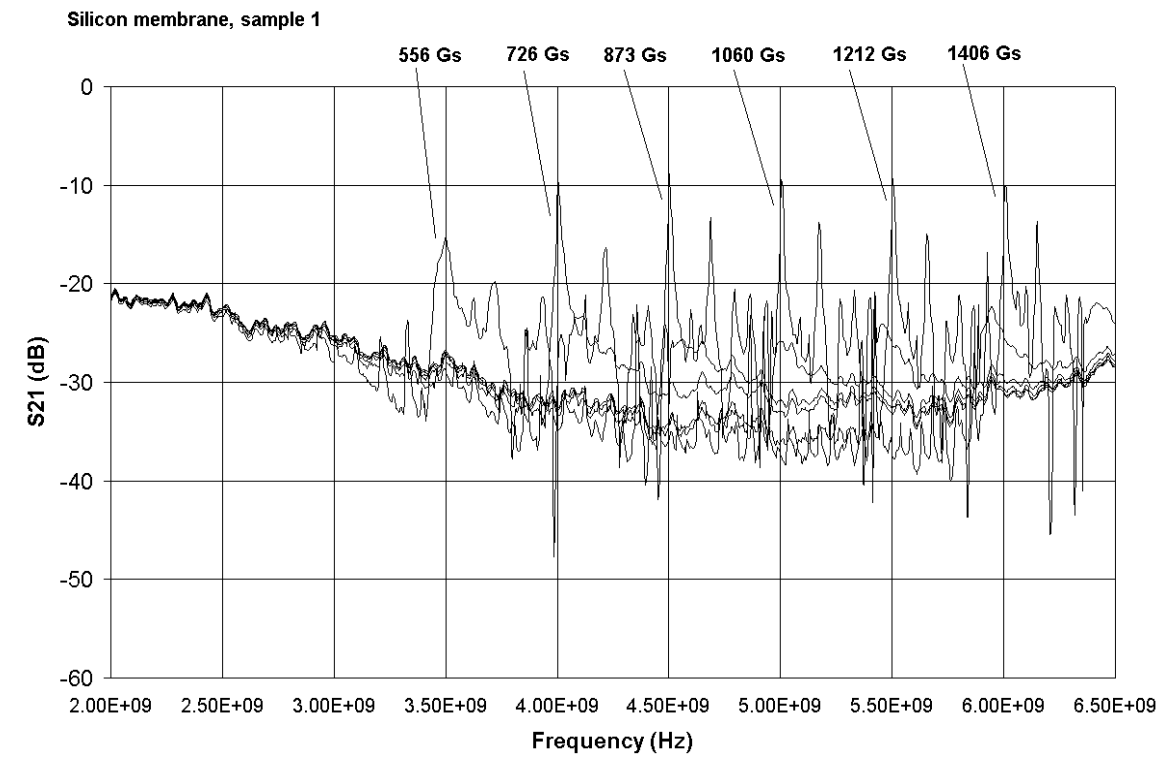

Fig. 4. Transmission characteristic for the MSW-SER on membrane, sample \#1.

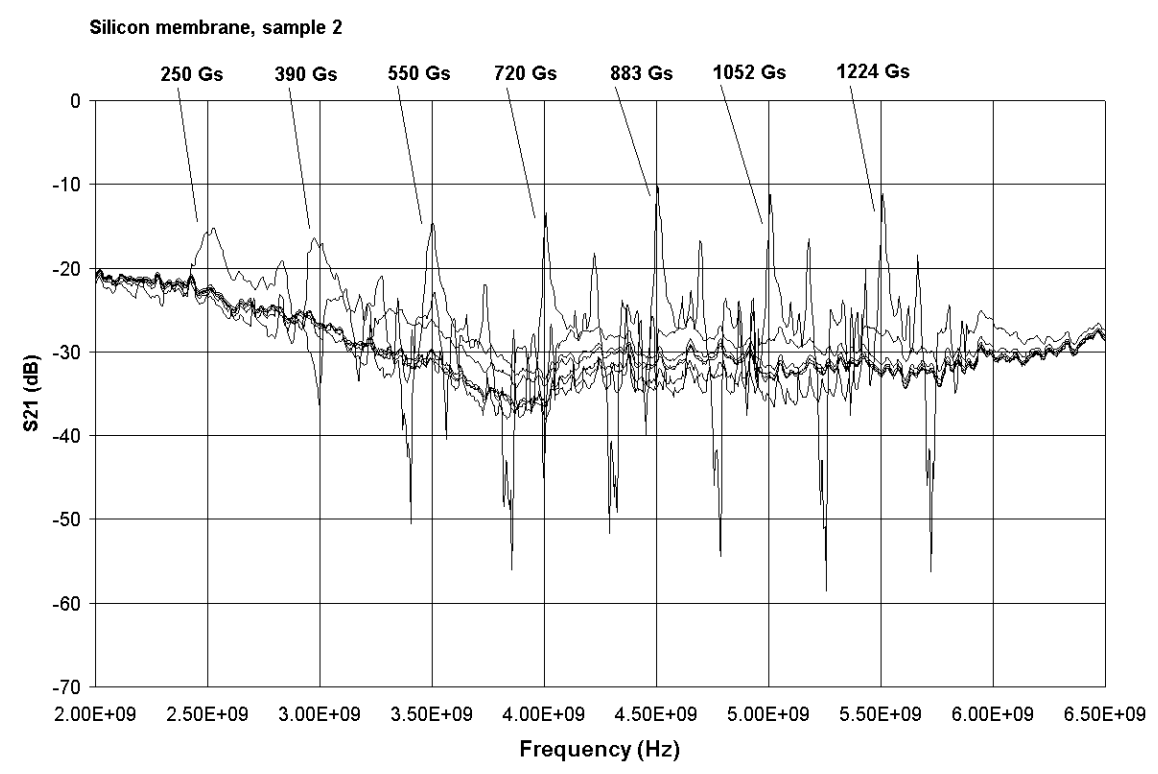

Fig. 5. Transmission characteristics for the MSW-SER on membrane, sample \#2. 